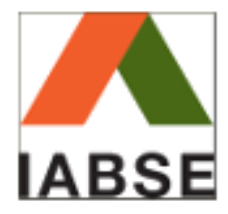

\title{
Structural Engineering
} International

\section{Maximum Likelihood Finite-Element Model Updating of Civil Engineering Structures Using Nature-Inspired Computational Algorithms}

\begin{tabular}{|r|l|}
\hline Journal: & Structural Engineering International \\
\hline Manuscript ID & Draft \\
\hline Manuscript Type: & Scientific Papers \\
\hline Type of Structure: & Bridges, Buildings, Dams \\
\hline Material and Equipment: & Composites, Steel, Bearings/Joints/Seismic Device \\
\hline Other Aspects: & $\begin{array}{l}\text { Dynamic effects / vibrations, Instrumentation / Monitoring, Assessment / } \\
\text { Repair }\end{array}$ \\
\hline
\end{tabular}

\section{SCHOLARONE \\ Manuscripts}




\section{FOCUS POINTS}

- Finite-Element Model Updating of Civil Engineering Structures is usually performed under the Maximum Likelihood Method.

- According to this method, the updating problem is transformed into a global optimization problem.

- This optimization problem can be formulated under two approaches: (i) singleobjective function approach; and (ii) multi-objective function approach.

- Nature-inspired computational algorithms, which allow finding efficiently the global solution of the problem but they require a high simulation time to tackle this problem, are usually employed to solve this optimization problem.

- Two are the main contributions of this study: (i) to establish which is the most adequate approach to formulate the updating problem; and (ii) to determine which is the most efficient NIC algorithm to solve this optimization problem.

- Harmony search has been implemented innovatively herein to cope with the FE model updating problem. 


\section{Maximum Likelihood Finite-Element Model Updating of Civil Engineering Structures Using Nature-Inspired Computational Algorithms}

Javier Fernando Jiménez-Alonso ${ }^{\mathrm{a}^{*}}$, Javier Naranjo-Perez ${ }^{\mathrm{b}}$, Aleksandar Pavic $^{c}$ and Andrés Sáez ${ }^{\mathrm{d}}$

$a^{*}$ Assistant professor. Department of Continuum Mechanics and Structures, E.T.S. Ingenieros de Caminos, Canales y Puertos. Universidad Politécnica de Madrid, Madrid (Spain).

${ }^{b}$ PhD Candidate. Department of Continuum Mechanics and Structural Analysis, Universidad de Sevilla, Seville (Spain).

${ }^{c}$ Full professor. Vibration Engineering, College of Engineering, Mathematics and Physical Sciences, University of Exeter, Exeter (U.K.).

${ }^{d}$ Full professor. Department of Continuum Mechanics and Structural Analysis, Universidad de Sevilla, Seville (Spain).

*Corresponding author: Assistant Professor: Javier Fernando Jiménez-Alonso. Department of Continuum Mechanics and Structures. E.T.S. Ingenieros de Caminos, Canales y Puertos. Universidad Politécnica de Madrid, Calle del Profesor Aranguren, 3, 28040 Madrid (Spain) Ph:+34 91 0674154. e-mail:jf.jimenez@upm.es. 


\title{
Maximum Likelihood Finite-Element Model Updating of Civil Engineering Structures Using Nature-Inspired Computational Algorithms
}

\begin{abstract}
Finite-element model updating of civil engineering structures is usually performed under the maximum likelihood method for practical engineering applications. According to this method, the updating problem may be transformed into an optimization problem. Thus, the value of the most relevant physical parameters of the model is obtained via the minimization of an objective function. This function is normally defined in terms of the differences between the numerical and experimental modal properties of the structure. Two different approaches may be used to define this objective function: (i) single-objective or (ii) multi-objective approach. Due to the complexity of the problem, global optimization algorithms are usually considered. Among these algorithms, natureinspired computational algorithms have been widely used for finite-elementmodel updating of civil engineering structures. Nevertheless, this method presents two main limitations: (i) a clear dependence between the updated model and the objective function considered for the updating problem; and (ii) a high simulation cost. In order to overcome these drawbacks, a detailed study has been performed herein both to find the most efficient computational algorithm among several well-known nature-inspired algorithms and to establish the most adequate objective function to tackle the updating problem. A laboratory footbridge has been considered as benchmark for this purpose.
\end{abstract}

Keywords: finite-element model updating, maximum likelihood method, singleobjective optimization, multi-objective optimization, nature-inspired computational algorithms, harmony search, civil engineering structures. 


\section{Funding}

This work was supported by the Ministerio de Economía y Competitividad of Spain and the European Regional Development Fund under project RTI2018-094945-BC21. The financial support is gratefully acknowledged. Additionally, the co-author, J. Naranjo-Pérez, has been supported by the research contract, USE-17047-G, provided by the Universidad de Sevilla. 


\title{
Maximum Likelihood Finite-Element Model Updating of Civil Engineering Structures Using Nature-Inspired Computational Algorithms
}

\begin{abstract}
Finite-element model updating of civil engineering structures is usually performed under the maximum likelihood method for practical engineering applications. According to this method, the updating problem may be transformed into an optimization problem. Thus, the value of the most relevant physical parameters of the model is obtained via the minimization of an objective function. This function is normally defined in terms of the differences between the numerical and experimental modal properties of the structure. Two different approaches may be used to define this objective function: (i) single-objective or (ii) multi-objective approach. Due to the complexity of the problem, global optimization algorithms are usually considered. Among these algorithms, natureinspired computational algorithms have been widely used for finite-elementmodel updating of civil engineering structures. Nevertheless, this method presents two main limitations: (i) a clear dependence between the updated model and the objective function considered for the updating problem; and (ii) a high simulation cost. In order to overcome these drawbacks, a detailed study has been performed herein both to find the most efficient computational algorithm among several well-known nature-inspired algorithms and to establish the most adequate objective function to tackle the updating problem. A laboratory footbridge has been considered as benchmark for this purpose.
\end{abstract}

Keywords: finite-element model updating, maximum likelihood method, singleobjective optimization, multi-objective optimization, nature-inspired computational algorithms, harmony search, civil engineering structures.

\section{Introduction}

Finite-element (FE) models have been extensively used to simulate numerically the behaviour of civil engineering structures. However, these numerical models do not frequently reflect adequately the actual behaviour of the structures, so they need to be improved based on the results obtained from field tests and continuous monitoring [1]. After the adjustment, these numerical models better characterize the real response of the structures under external actions. For practical engineering applications, the tuning of the numerical models is usually achieved via the modification of the most relevant physical parameters of the structure in order to reduce the differences between its numerical and experimental behaviour. After the updating process, the resulting updated 
model can be used either to assess more accurately the behaviour of civil engineering structures $[2,3,4]$ or to establish a strategy for damage detection $[5,6]$.

As one of the main objectives of the FE model updating is to estimate indirectly the value of the most relevant physical parameters of the structure, the updating problem can be formulated as a parameter identification problem [7]. In order to solve the parameter identification problem, the so-called estimators are usually employed [8]. Estimators can be classified into two general groups [9, 10]: (i) point estimators, which return the expected value of each considered design parameter; and (ii) interval estimators, which return either an interval in which the value of each considered design parameter may be located or even the probability density function of each considered design parameter. On the one hand, among the point estimators, the maximum likelihood method (MLM) [8] has been widely used due to its efficiency and accuracy to solve the updating problem for practical engineering applications [11]. On the other hand, among the interval estimators, the Bayesian method [12] has imposed since it allows estimating numerically the probabilistic density function of the considered design parameters which can be useful to perform any kind of structural reliability analysis [13].

Among these two methods, the MLM has been commonly employed for practical engineering applications due to: (i) an easier implementation, (ii) the lower simulation time required to solve the updating problem (against the Bayesian method); and (iii) the straightforward use of the updated models for deterministic applications [2, 11]. For all these reasons, this study focuses on the analysis of the performance of FE model updating under the MLM

The parameter identification problem, under the MLM and assuming a normal distribution of the adjustment errors, is equivalent to the ordinary least squares problem [14]. Thus, under this assumption, the parameter identification problem may be transformed into an optimization problem, and therefore the model updating problem may be formulated as an optimization problem. The purpose of this optimization problem is to obtain the value of the design parameters of the structure which minimizes the difference between the numerical and experimental behaviour of the structure. In order to characterize the behaviour of the structure, its modal properties are normally considered [8]. Thus, the numerical modal properties of the structure are commonly obtained from a modal analysis based on the FE method [8]; and the experimental modal properties are identified via the signal processing (either experimental or operational modal analysis) of the records obtained during an experimental test (either forced or ambient vibration test) [15].

In this manner, the objective function of this optimization problem can be defined in terms of the relative differences (residuals) between the numerical and experimental modal properties of the structure. As two types of modal properties are normally considered (natural frequencies and associated vibration modes), two type of residuals must be taken into account for the definition of the objective function. As these modal properties have different nature, a new problem arises, how to weigh the influence of each residual on the definition of the objective function. Two approaches are considered to cope with this problem [16]: (i) the single-objective function (SOF) approach; where a single objective is optimized and different weighting factors are considered to assess the influence of each residuals; and (ii) the multi-objective function (MOF) approach, where the different terms of a multi-objective function are optimized which are defined in terms of each residuals. Both approaches have been used interchangeably for practical engineering application [17, 18], being rare the studies in 
which the performance of these two approaches has been compared. For this reason, one of the objectives of this study is to shed light to this issue.

On the other hand, an optimization algorithm must be selected to solve this minimization problem. Due to the nonlinear relationship between the modal properties of the structure and the considered physical parameters, computational intelligence algorithms are usually considered for this purpose [8]. Computational algorithms allow finding the global optimum of nonlinear optimization problems regardless of the initial searching point considered. Among these computational algorithms, nature-inspired computational (NIC) algorithms have been widely employed to solve the FE model updating problem [8]. Nevertheless, these computational algorithms present a clear drawback, the high simulation time required to perform the updating process. In order to overcome this limitation, it must be checked which is the most efficient NIC algorithm to solve each particular optimization problem. Herein, a comparison study will be carried out to analyse the performance of several NIC algorithms to solve the updating problem.

Therefore two are the main objectives of this study: (i) to establish which is the most adequate approach, either SOF approach or the MOF approach, to formulate the updating problem under the MLM; and (ii) to determine which is the most efficient NIC algorithm to solve the FE model updating problem among three different computational algorithms.

Three NIC algorithms have been compared herein: (i) genetic algorithms (GA); the most commonly employed computational algorithm to cope with the updating problem [19]; (ii) the particle swarm (PS) algorithm, which has shown a great efficiently to solve the FE model updating problem of mechanical structures [20]; and (iii) the harmony search (HS) algorithm, a more recent global optimization algorithm [21], that although it has been applied successfully for structural engineering applications [22], however, it has not been yet implemented for the FE model updating of civil engineering structures.

For this purpose, the performance of the two mentioned approaches and the three considered NIC algorithm has been compared when they are implemented to perform the FE model updating of a real civil engineering structure. As benchmark structure, a laboratory steel footbridge located at the Vibration Engineering Section of the University of Exeter (U.K.) has been considered [23]. A FE model of the structure has been built. The numerical modal properties of the structure have been obtained via a modal analysis. Subsequently, the experimental modal properties have been identified experimentally via an experimental modal analysis. The records obtained in a forced vibration test have been used for this purpose. A bad correlation between the numerical and experimental modal properties has been computed and the necessity of performing a FE model updating has been revealed. In order to compare the different model updating processes, three comparison criteria have been considered to determine the most adequate approach for FE model updating of civil engineering structures under the MLM; the sweep capacity, the computational cost and the goodness of the solution, and other two comparison criteria have been taken into account for the determination of the most efficient NIC algorithm; the computational cost and the accuracy of the adjustment.

The results of this study can be implemented easily to improve the FE model updating of real civil engineering structures for practical engineering applications. Nevertheless, further studies are needed to assess the performance of these NIC 
algorithms when they are implemented for the FE model updating of different types of civil engineering structures under the MLM.

The paper is organized as follows. Some basics of the FE model updating problem under the MLM are described in second section. Third section is devoted to the description of the three NIC algorithms considered herein. In fourth section, a real casestudy is presented. The FE model updating of a steel laboratory footbridge is performed under the two mentioned approaches and the three considered NIC algorithms. A discussion about the comparison of the results obtained is also included. Finally, some concluding remarks are drawn to close the paper in last section.

\section{Basics of FE Model Updating under the Maximum Likelihood Method}

According to the MLM, the updating process consists in the modification of the most relevant physical parameters of the FE model in order to adjust the numerical estimation of the response of the structure to its actual structural behaviour [14]. Hence, according to this method, the updating problem may be transformed into an optimization problem [8].

Therefore, the main objective of this optimization problem is to obtain the value of some design parameters which minimizes the value of an objective function defined in terms of the relative differences (residuals) between the numerical and experimental modal properties of the structure [8]. As design parameters, the physical parameters, which have the greatest influence on the dynamic behaviour of the structure, are usually selected [8]. The search space is normally constrained by a search domain to guarantee that the solution obtained maintains the physical meaning of the problem.

Two types of residuals are usually considered to define the objective function of the updating problem: (i) residuals associated with the natural frequencies, $r_{j}^{f}$ (being $j$ the considered natural frequency), and (ii) residuals associated with the vibration modes, $r_{j}^{m}$ (being $j$ the considered vibration mode).

The influence of these two types of residuals in the objective function is evaluated via two different approaches: (i) the single-objective function (SOF) approach; and (ii) the multi-objective function (MOF) approach.

Under the SOF approach, the objective function is defined in terms of the weighted residuals between the numerical and experimental modal properties of the structure. These weights are established to consider the relative contribution of each residual. Their value can be determined either by a statistical criterion [1] or by a trialand-error criterion [5]. According to the statistical criterion, the weights are defined in terms of the uncertainty associated with the estimates of the experimental modal properties of the structure. According to the trial-and-error criterion, the weights are defined by an iterative process which ensures the best agreement between the numerical and experimental modal properties of the structure. Herein the performance of the second criterion has been analysed.

Under the MOF approach, the objective function is defined in terms of several functional components. For practical engineering applications, this objective function is defined by two functional components [16]: (i) the first component is defined in terms of the residuals associated with the natural frequencies, $r_{j}^{f}(\boldsymbol{\theta})$; and (ii) the second component is defined in terms of the residuals associated with the vibration modes, $r_{j}^{m}($ $\boldsymbol{\theta}$ ) (being $j$ the considered vibration mode). Although, this approach presents as 
advantage that it is not necessary to define the weights of the residuals, it has a clear drawback, a subsequent decision-making problem must be solved to select the best solution among the set of possible solutions (the so-called Pareto front) provided by the optimization algorithm. In order to solve this decision-making problem, the normal boundary intersection (NBI) method has been considered herein [24] among the different criteria provide in literature [25].

Thus, the general formulation of the FE-model updating problem under the SOF approach may be stated as follows [16]:

$$
\begin{aligned}
& \min f(\boldsymbol{\theta})=\min \sum_{j=1}^{m} w_{j} r_{j}(\boldsymbol{\theta})^{2}=\min \sum_{j=1}^{m_{f}} w_{j}^{f} r_{j}^{f}(\boldsymbol{\theta})^{2}+\sum_{j=1}^{m_{m}} w_{j}^{m} r_{j}^{m}(\boldsymbol{\theta})^{2} \\
& \boldsymbol{\theta}^{l} \leq \boldsymbol{\theta} \leq \boldsymbol{\theta}^{u} \quad \sum w_{j}=\sum w_{j}^{f}+w_{j}^{m}=1 \quad w_{j} \geq 0
\end{aligned}
$$

where $f(\boldsymbol{\theta})$ is the objective function for the single-objective function approach (SOF); $m_{f}$ is the size of the residuals vector related to the natural frequencies, $\mathbf{r}^{\mathbf{f}}(\boldsymbol{\theta}) ; m_{m}$ is the size of the residuals vector related to the vibration modes, $\mathbf{r}^{\mathbf{m}}(\boldsymbol{\theta}) ; m$ is the size of the residuals vector, $\mathbf{r}(\boldsymbol{\theta}) ; w_{j}$ is the weighting factor of the element $j$ of the residuals vector; $r_{j}(\boldsymbol{\theta})$ is the element $j$ of the residuals vector; $w_{j}^{f}$ is the weighting factor of the element $j$ of the residuals vector associated with the natural frequencies, $r_{j}^{f}(\boldsymbol{\theta})$ is the element $j$ of the residuals vector associated with the natural frequencies; $w_{j}^{m}$ is the weighting factor of the element $j$ of the residuals vector associated with the vibration modes; $r_{j}^{m}(\boldsymbol{\theta})$ is the element $j$ of the residuals vector associated with the vibration modes; $\boldsymbol{\theta}=\left[\theta_{1}, \ldots, \theta_{i}\right.$ $\left., \ldots, \theta_{n_{v}}\right]$ is the physical parameter vector/design parameter vector (with $n_{v}$ the number of terms of this vector); $\boldsymbol{\theta}^{l}=\left[\theta_{1}^{l}, \ldots, \theta_{i}^{l}, \ldots, \theta_{n_{v}}^{l}\right]$ is the lower bound vector of the search domain of the physical parameter vector; and $\boldsymbol{\theta}^{u}=\left[\theta_{1}^{u}, \ldots, \theta_{i}^{u}, \ldots, \theta_{n_{v}}^{u}\right]$ is the upper bound vector of the search domain of the physical parameter vector.

On the other hand, the general formulation of the FE model updating problem under the MOF approach may be stated as follows [16]:

$$
\begin{aligned}
& \min \mathbf{f}(\boldsymbol{\theta})=\left(\begin{array}{ll}
f_{1}(\boldsymbol{\theta}) & f_{2}(\boldsymbol{\theta})
\end{array}\right)=\min \left(\sum_{j=1}^{m_{f}} r_{j}^{f}(\boldsymbol{\theta})^{2} \quad \sum_{j=1}^{m_{m}} r_{j}^{m}(\boldsymbol{\theta})^{2}\right) \\
& \boldsymbol{\theta}^{l} \leq \boldsymbol{\theta} \leq \boldsymbol{\theta}^{u}
\end{aligned}
$$

where $f_{1}(\boldsymbol{\theta})$ and $f_{2}(\boldsymbol{\theta})$ are respectively the first and the second sub-objective functions for the multi-objective function approach.

In order to guarantee that the two types of residuals are well-balanced, the residuals may be normalized as follows [16]:

$$
\begin{gathered}
r_{j}^{f}(\boldsymbol{\theta})=\frac{f_{\text {num }, j}(\boldsymbol{\theta})-f_{\text {exp }, j}}{f_{\text {exp }, j}} j=1,2, \ldots, m_{f} \\
r_{j}^{m}(\boldsymbol{\theta})=\sqrt{\left(\frac{\left(1-\sqrt{M A C_{j}(\boldsymbol{\theta})}\right)^{2}}{M A C_{j}(\boldsymbol{\theta})}\right)} j=1,2, \ldots, m_{m}
\end{gathered}
$$

Therefore the FE model updating process consists of the following steps [8]: (i) the modal properties of the structure are identified experimentally via the signal 
processing of the records obtained during a vibration test; (ii) the numerical modal properties of the structure are obtained via a FE modal analysis; (iii) the objective function is evaluated; (iv) the design parameters are modified; and (v) the steps (ii) to (iv) are repeated iteratively until some convergence criterion is met.

As result of this process, either a vector of updated design parameters (SOF approach) or the Pareto front (MOF approach) is obtained. Additionally, a subsequent decision-making problem must be tackled to select the vector of updated parameters under the MOF approach. Fig. 1 shows the flowchart of the FE model updating problem under the MLM.

Fig. 1. Flowchart of the FE model updating problem under the MLM [26].

In order to assess the performance of the updating process, the correlation between the numerical and experimental modal properties must be determined. For this purpose, several ratios have been proposed [15]. Among the different proposals, the relative difference between the numerical and experimental natural frequencies, $\Delta f_{\text {exp,j }}^{n u m, j}$ $(\boldsymbol{\theta})$, and the modal assurance criterion, $M A C_{\text {exp,j }}^{\text {num } j}(\boldsymbol{\theta})$, are widely used [1]. Both ratios are defined as:

$$
\begin{gathered}
\Delta f_{\text {exp }, j}^{n u m}(\boldsymbol{\theta})=\frac{f_{\text {num }, j}(\boldsymbol{\theta})-f_{\text {exp }, j}}{f_{\text {exp }, j}} 100[\%] \\
M A C_{\text {exp }, j}^{n u m, j}(\boldsymbol{\theta})=\frac{\left(\phi_{n u m, j}^{T}(\boldsymbol{\theta}) \cdot \phi_{\text {exp }, j}\right)^{2}}{\left(\phi_{\text {num }, j}^{T}(\boldsymbol{\theta}) \cdot \phi_{\text {num }, j}(\boldsymbol{\theta})\right)\left(\phi_{\text {exp }, j}^{T} \cdot \phi_{\text {exp }, j}\right)}
\end{gathered}
$$

where $f_{\text {num }, j}(\boldsymbol{\theta})$ and $f_{\text {exp,j }}$ are respectively the numerical and experimental $j$ natural frequencies of the structure; $\phi_{n u m, j}(\boldsymbol{\theta})$ and $\phi_{\text {exp }, j}$ are the numerical and experimental $j$ vibration modes; and ${ }^{T}$ denotes the transpose function.

A good correlation between the numerical and experimental modal properties of the structure is achieved if both the relative differences, $\Delta f_{\text {exp,j }}^{n u m}(\boldsymbol{\theta})$, are lower than $5.00 \%$ and their corresponding $M A C_{\text {exp, }, j}^{n u m}(\boldsymbol{\theta})$ ratios are greater than 0.90 [27].

\section{Nature-Inspired Computational Algorithms for Numerical Optimization}

Nature-inspired computational (NIC) algorithms have been widely used to solve the FE model updating problem under the MLM. The main advantages of these computational algorithms are: (i) their independence in relation to the initial values of the design parameters; and (ii) their ability to find the global optimum of the objective function. However, they present a clear drawback; the high simulation time required to solve the updating problem. For this reason, it is necessary to assess the performance of each NIC algorithm when it is implemented in a particular optimization problem.

For this purpose, the performance of three NIC algorithms, under both the SOF approach and the MOF approach, has been compared herein: (i) genetic algorithms (GA); (ii) particle swarm (PS) algorithm; and (iii) the harmony search (HS) algorithm. These NIC algorithms are described briefly in this section.

\section{Genetic Algorithms (GA)}

The genetic algorithms (GA) are a NIC algorithms based on the Darwin's natural selection theory [19]. According to this theory, each possible value of the design parameters is identified as a chromosome. Thus, each set of design parameters is 
grouped into an individual (parameter vector). Subsequently, the value of this parameter vector is improved via an iterative process where the value of the objective function is optimized. The GA can be summarized in the following steps: (i) an initial random population of parameter vectors is created; (ii) the objective function is evaluated for all the individuals; (iii) a new population is created using three mechanisms (selection, crossover and mutation); (iv) the objective function is evaluated for the individuals of the new population; (v) the steps (iii) and (iv) are repeated until some convergence criterion is met. Fig. 2a shows the flowchart of the GA.

Three mechanisms are used for this algorithm to find the optimum value of the objective function: (i) the selection, where a part of the initial population is maintained when a new population is generated; (ii) the crossover, where a new parameter vector is derived from two previous ones; and (iii) the mutation, where a new parameter vector is generated from a previous one modifying randomly the value of one element of the vector. The combination of these two last mechanisms allows sweeping the search domain of the optimization problem. Three design variables are defined to characterize these three mechanisms: (i) the selection ratio, selr, which establishes the number of individuals that constitute the elite of the population, they are maintained in the next generation and they are used as parents for the generation of new individuals using the crossover and mutation mechanism; (ii) the crossover ratio, crosr, which determines the percentage of the remaining population that is modified by crossover mechanism; and (iii) the mutation ratio, mutr, which establishes the percentage of the remaining population that is modified by the mutation mechanism.

\section{Particle Swarm (PS) Algorithm}

The particle swarm (PS) algorithm was proposed by Kennedy and Eberhart [20]. It is a NIC algorithm based on social behaviour of biological organisms when they move in a single group to achieve a desired overall objective.

According to this, the particles move through the search space following simple laws related to their position (value of the physical parameters) and velocity. The position of each particle is improved in terms of both its own best position and the best position of the remaining particles. Thus, as the position of each particle is updated in terms of its velocity, the performance of the algorithm depends mainly on this design variable.

Hence, the particle's velocity depends on three main parameters: (i) the particle's inertia, ipar, which controls the impact of the current velocity on the subsequent velocity; (ii), a self-adjustment weight, selw; which weighs the influence of the best position achieved by this particle on the subsequent velocity; and (iii) a socialadjustment weight, socw, which weighs the influence of the best position achieved by the best particle in the swarm on the subsequent velocity. The particle's inertia, ipar, controls the exploratory properties of the simulation. Thus, a high value of inertia encourages a global exploration, while a low value of the inertia encourages local exploration. The self-adjustment weight, selw, indicates the confidence that the current particle has on itself and the social-adjustment weight, socw, indicates the confidence the current particle has on the successes of the population [8]

The PS algorithm consists of the following steps: (i) a population of particles' position and velocities are generated randomly (the particles' position must be distributed in the search domain); (ii) the velocity of each particle is evaluated; (iii) the 
position of each particle is updated; (iv) steps (ii) and (iii) are repeated until some convergence criterion is met. Fig. 2b shows the flowchart of the PS algorithm.

\section{Harmony Search (HS) Algorithm}

The HS algorithm was initially formulated by Zong Woo Geem et al. [21]. It is based on the improvisation carried out by the musicians when they are searching for the harmony following aesthetic requirements. The HS algorithm consists of the following steps: (i) an initial random population of possible solutions, which is stored in the harmony memory matrix, HM, is created; (ii) the objective function is evaluated for each of the candidate solutions; (iii) a new harmony is created at each iteration; (iv) the objective function is evaluated for each new harmony, (v) the harmony memory matrix, HM, is updated comparing the original and new harmonies; and (vi) the steps (iii) to (v) are repeated until some convergence criterion is met. Fig. $2 \mathrm{c}$ also shows the flowchart of the HS algorithm.

For the generation of a new harmony, three mechanisms are considered: (i) harmonies in memory; (ii) pitch adjustment; and (iii) randomization. Each parameter from a new vector may be defined either from a previous value stored in the harmony memory matrix, HM, or from a new random value. These two possibilities are controlled by the harmony memory consideration rate, HMCR which establishes the probability of selecting a previous element of the harmony memory matrix, HM.. Additionally, if the value is adopted from a previous one, it can be mutated according to the pitch adjustment rate, $P A R$, and considering a predefined bandwidth, $b w$, of possible values.

Fig. 2. Flowchart of the three considered NIC algorithms: a) genetic algorithms (GA); b) particle swarm (PS); and c) harmony search (HS).

\section{MOF Approach for the Three Considered NIC Algorithms}

The MOF approach of the GA is implemented via the NSGA-II (Non-dominated Sorting Genetic Algorithm) [28]. This algorithm makes use of the non-dominated sorting technique [29] to distinguish between dominated and non-dominated (those that improve the value of one objective function without sacrificing the others) solutions. For this purpose, each individual (possible solution) is characterized by an operator socalled, crowded comparison operator. This operator takes into account two attributes of the solution: (i) the rank; and (ii) the crowding distance.

The steps of the GA under the MOF approach are: (i) an initial population is randomly created; (ii) the multi-objective function is evaluated for each individual; (iii) subsequently, the non-dominated sorting technique is employed to sort the solutions based on the rank and the crowding distance; (iv) then an iterative process is initialized where a new population is generated using both the crossover and mutation mechanisms; (v) the multi-objective function is evaluated for the new population and the non-dominated sorting technique is used again; (vi) the initial size of the population is restored via its truncation based on the worst crowding distance solutions; and (vii) the steps (iv) to (vi) are repeated until some convergence criterion is met. As result of this process, the so-called Pareto front, a set of possible solutions of the updating problem, is obtained.

Subsequently, the MOF approach of the PS algorithm is also based on the classification of the non-dominated particles [30]. Hence, once the initial particles' population is created and the multi-objective function is evaluated, the non-dominated solutions are obtained and stored in the particles' repository. A similar process, to 
define the Pareto front, is obtained after the mutation of the position. Thus, if the mutated particle dominates the non-mutated, the latter is updated. This step is applied iteratively to both the updated particle's position and the best particle's position. Then, the non-dominated solutions are added to the repository and finally, the domination is evaluated for the overall repository to neglect the dominated particles. These steps are repeated iteratively until some convergence criterion is met. As results of this process, the Pareto font is obtained.

Finally, the MOF approach of the HS algorithm has also been implemented [31] considering the main rules of the NSGA-II algorithm. According to this, the HS algorithm under a MOF approach consists of the following steps: (i) an initial set of harmonies is generated (harmony memory matrix, HM); (ii) the multi-objective function is evaluated for all of harmonies; (iii) the attributes of each individual are calculated using the crowded comparison operator; (iv) the harmonies are sorted based on the crowding distance; (v) a new set of harmonies is created based on the three mentioned design variables (HMCR, PAR and $b w)$; (vi) the multi-objective function is evaluated for the new harmonies; (vii) the harmony memory matrix, HM, is truncated using both the non-dominated sorting technique and the crowding distance; and (viii) the steps (v) to (vii) are repeated until some convergence criterion is met. Finally, as result of this process, the Pareto front is obtained.

\section{Discussion about the Performance of the Three Considered Nature-Inspired Algorithms}

As Fig. 2 illustrates, the three considered NIC algorithms under the SOF approach share three common characteristics: (i) the random initialization of a population; (ii) the implementation of several mathematical operations to modify this population; and (iii) the iterative application of this re-generation process until some convergence criteria is met. Additionally, they also share the use of the non-dominated sorting rules for their implementation under a MOF approach.

However, some particular differences among these NIC algorithms make interesting comparing their performance when they are implemented for the FE model updating of civil engineering structures.

Between the GA and the HS algorithm, the main difference lies in the different complexity of the operations which control the updating of the population. Therefore, the operations associated with the HS algorithm (selection, improvement and randomization) are computationally easier than the operations associated with the GA (selection, crossover and mutation). Thus, it is expected that the computational time required to solve an optimization problem using the HS algorithm is lower than the one required employing GA. However, the simplicity of the operations can have a negative influence on both the accuracy of the solution and the sweeping capacity of the algorithm, reducing the performance of the HS algorithm when it is implemented for a practical engineering application.

Additionally, among these two algorithms (GA and HS) and the PS algorithm, the main difference is related to the re-generation of the population. While the two mentioned algorithms (GA and HS) generate iteratively different populations (new populations) during the updating process; the PS algorithm maintains the initial population and it only updates iteratively its attributes. In this manner, the PS algorithm may reduce the simulation time required to solve an optimization problem. However, as on the one hand, the sweeping capacity of the algorithm is lower than the GA; and on the other hand, the computational operations needed to compute the position and the velocity of each particle are more complex than the re-generating operations of the HS 
algorithm, it is not possible establish that the PS algorithm is the most efficient computational algorithm among the three considered ones to solve any optimization problem.

For all these reason, it is not possible to establish preliminary which is the most efficient algorithm to solve a particular optimization problem, being necessary to perform a comparative study to analyse their performance for each considered case. For this purpose, the performance of the three mentioned NIC algorithms, when they are implemented for the FE model updating of a real case-study, have been compared in next section.

\section{Application Example: FE Model Updating of a Laboratory Footbridge}

A real case-study, an application example, has been analysed to meet the two objectives of this study: (i) to establish the most adequate approach to formulate the updating problem under the MLM; and (ii) to determine the most efficient NIC algorithm among the three above mentioned computational algorithms. Therefore, the performance of the three mentioned NIC algorithms under the two approaches has been compared when they have been implemented to perform the FE model updating of a laboratory structure. The comparison process is described in detail in this section.

\section{Preliminary FE Model and Numerical Modal Analysis of the Laboratory Footbridge}

This laboratory footbridge is a frame structure with a single span of $15 \mathrm{~m}$. The structure is configured by two lateral steel beams separated transversally $2.5 \mathrm{~m}$. These beams are braced by diaphragms separated longitudinally $1.25 \mathrm{~m}$. The diaphragms consist in rectangular plates of $200 \times 12 \mathrm{~mm}$. The lateral beams are connected in their both sides to steel columns. These columns are pinned directly to the ground. The deck of the structure is formed by composite SPS panels [32]. These composite SPS panels are bolted to both the longitudinal and transversal elements. An overall view of the structure and its main constitutive components is illustrated in Fig. 3. A more detailed description of this structure can be found in Ref [23].

The structure was modelled with three types of elements in the FE package Ansys [33]. All the numerical simulations were performed using a laptop computer with a processor of $3.6 \mathrm{GHz}$ and a RAM memory of $8 \mathrm{~GB}$. The mesh consisted of 31903 elements (Fig. 5). For our purpose the FE model was performed according to the following scheme: (i) the two main lateral beams and the transversal plates have been modelled with a four node shell elements with six degrees of freedom at each node (SHELL181); (ii) the SPS panels have been modelled with the same type of element (SHELL181) considering its sandwich behaviour through the first-order sheardeformation theory; (iii) the bolts that configure the connection between the SPS panel and the steel structure have been modelled by 3-D beam elements (BEAM188); and (iv) the supports were modelled by means of longitudinal and lateral spring elements (COMBIN14) and considering that the vertical displacement was constrained.

The initial value for these spring parameters has been estimated from a simplified FE model of just the column that configure the support, resulting an equivalent stiffness of $k_{\text {lon }}=5.5 \cdot 10^{7} \mathrm{~N} / \mathrm{m}$ in the longitudinal direction and $k_{\text {lat }}=1.9$. $10^{7} \mathrm{~N} / \mathrm{m}$ in the lateral direction.

As mechanical properties of the constitutive materials the following values were considered: (i) for the steel [34], a density $\gamma_{s}=7850 \mathrm{~kg} / \mathrm{m}^{3}$, a Young's modulus $E_{s}$ $=2.1 \cdot 10^{5} \mathrm{MPa}$ and a Poisson's ratio $v_{s}=0.3$; and (ii) for the polyurethane [35], a 
density $\gamma_{p}=1100 \mathrm{~kg} / \mathrm{m}^{3}$, a Young's modulus $E_{p}=750 \mathrm{MPa}$ and a Poisson's ratio $v_{p}$ $=0.5$.

This FE has been used to perform a numerical modal analysis. The first seven natural frequencies, $f_{\text {num, }}(\boldsymbol{\theta})$, and associated numerical vibration modes, $\phi_{\text {num, }}(\boldsymbol{\theta})$, have been obtained from this numerical analysis (being $j$ the considered vibration mode). Thus, Table 1 shows the first seven numerical natural frequencies, $f_{\text {num, }, j}(\boldsymbol{\theta})$, and Fig. 3 illustrates the first seven numerical vibration modes, $\phi_{\text {num }, j}(\boldsymbol{\theta})$.

Fig. 3. Description of the structure and initial first seven numerical vibration modes, $\phi_{\text {num }, j}(\boldsymbol{\theta})$, being $j$ the considered vibration mode.

\section{Experimental Identification of the Modal Properties of the Laboratory Footbridge}

Subsequently, a forced vibration test was conducted to identify experimentally the modal properties of the footbridge. A set of proof-mass actuators and rowing accelerometers were used for this purpose (Fig. 4). The actuators were simultaneously driven by uncorrelated random signals considering a Multi-input Multi-output (MIMO) configuration [15, 23]. At all the considered reference points (instrumented points), the Frequency Response Functions (FRFs) were determined considering an overlap of 50\%. Later, probable mode locations were identified in the FRFs fitted curves via a complex mode indicator function. Finally, a global polynomial curve fitting method has been used to identify experimentally the first seven natural frequencies and associated vibration modes based on the mentioned probable mode locations [15]. Both the forced vibration test and the corresponding experimental modal analysis have been described in detail in Ref [23].

Fig. 4. Forced vibration test of the laboratory footbridge [23].

The first seven experimental natural frequencies, $f_{\text {exp,j, }}$, and associated experimental vibration modes, $\phi_{\text {exp }, j}$, were obtained as result of this experimental modal analysis (being $j$ the considered vibration mode). Thus, Table 1 shows the first seven experimental natural frequencies, $f_{\text {exp,j, }}$, and Fig. 5 illustrates the first seven experimental vibration modes, $\phi_{\text {exp }, j}$.

Correlation between the Preliminary Numerical and Experimental Modal Properties of the Laboratory Footbridge

In order to analyse the correlation between the numerical and experimental modal properties of the laboratory footbridge, the relative differences, $\Delta f_{\text {exp, }, j}^{n u m}(\boldsymbol{\theta})$, and the $\operatorname{MAC}(\boldsymbol{\theta})_{\text {exp,j }}^{\text {num } j}$ ratios were determined. Table 1 shows the correlation between the numerical and experimental modal properties of the laboratory footbridge. Additionally, Fig. 5 illustrates a comparison between the numerical, $\phi_{\text {num }, j}(\boldsymbol{\theta})$, and experimental, $\phi_{\text {exp }, j}$, vibration modes.

Table 1. Correlation between the initial numerical and experimental modal properties of the laboratory footbridge (being $f_{n u m, j}(\boldsymbol{\theta})$ the numerical natural frequencies; $f_{\text {exp }, j}$ the experimental natural frequencies; $\Delta f_{\text {exp,j }}^{n u(\boldsymbol{\theta})}$, the relative differences between the numerical and experimental natural frequencies; and the $M A C(\boldsymbol{\theta})_{\exp , j}^{n u m}$ ratio of the $j$ considered vibration mode).

As Table 1 shows that although the shapes of six of the seven identified vibration modes were in good agreement (with $M A C(\boldsymbol{\theta})_{\text {exp,j }}^{n u m}$ ratios greater than 0.90 ), 
the relative differences, $\Delta f_{\text {exp,j }}^{n u m}(\boldsymbol{\theta})$, between the numerical and experimental natural frequencies of four of the seven vibration modes were still large.

Therefore, it becomes necessary to improve the FE model of this structure in order to achieve a better correlation between its numerical and experimental modal properties and, consequently, to obtain a FE model which better reflects the actual dynamic behaviour of this structure [5].

\section{FE Model Updating of the Laboratory Footbridge}

In order to formulate the FE model updating problem of this laboratory footbridge under the MLM, the following elements must be defined: (i) the objective function of the problem (either $f(\boldsymbol{\theta})$ or $\boldsymbol{f}(\boldsymbol{\theta})$ ); (ii) the design parameters, $\boldsymbol{\theta}$; (iii) the search domain ( $\boldsymbol{\theta}^{\boldsymbol{l}}$ and $\boldsymbol{\theta}^{\boldsymbol{u}}$ ); and (iv) the optimization algorithm (GA, PS or HS).

Thus, the two approaches of the objective function have been defined in the second section; the considered optimization algorithms have been described in the third section; and the design parameters and their corresponding search domains have been established here.

Hence, set with the most relevant physical parameters of the model has been selected, $\boldsymbol{\theta}$. The modal strain energy associated with each physical parameter was considered as selection criterion [36]. A sensitivity analysis was carried out for this purpose. This analysis consists in calculating the ratio between the modal strain energy associated with each parameter and the overall modal strain energy of the structure. This ratio allows selecting the most relevant physical parameters for each considered vibration mode. After this sensitivity analysis, ten physical parameters of the structure were selected as design parameters. Table 2 and Fig. 7 illustrate the ten considered physical parameters. Additionally, a search domain has been included to constrain this optimization problem and thus, to guarantee the physical meaning of the updated model. Table 2 also shows the lower, $\boldsymbol{\theta}^{\mathbf{l}}$, and the upper, $\boldsymbol{\theta}^{\mathbf{u}}$, bounds of this search domain.

Fig. 5. FE model and comparison between the numerical, $\phi_{n u m, j}(\boldsymbol{\theta})$, and experimental, $\phi_{\text {exp, }}$, vibration modes of the laboratory footbridge (being $j$ the considered vibration mode).

Table 2. Search domain (lower bound, $\boldsymbol{\theta}^{\mathbf{l}}$, and upper bound, $\boldsymbol{\theta}^{\mathbf{u}}$ ) of the considered physical parameters of the model, $\boldsymbol{\theta}$.

After selecting both the design parameters and their corresponding search domain, the updating problem has been solved considering the two approaches and the three considered NIC algorithms.

\section{Comparison among GA, PS and HS for FE Model Updating under the SOF Approach}

First, the initial FE model of the laboratory footbridge was updating under the SOF approach considering the three mentioned NIC algorithms (GA, PS and HS). The solution of each updating problem has been performed via its implementation in both the FE analysis package Ansys [33] and the mathematical package Matlab [37]. Two comparison criteria were considered to validate the performance of the three considered NIC algorithms: (i) the computational cost; and (ii) the accuracy of the adjustment.

For the three NIC algorithms, the following common design variables were considered: (i) as population size, $P o p=100$; (ii) as maximum number of iterations, Iter $=50$; and (iii) as objective function tolerance, TolF $=10^{-5}$ (according to this, the 
algorithm stops if the average relative change of the best value of the objective function is less than or equal to TolF).

Additionally, for each NIC algorithms, the following particular design variables were considered: (i) for the GA, as selection ratio, selr $=0.1$, as crossover ratio, crosr $=0.9$, and as mutation ratio, mutr $=0.1$; (ii) for the PS algorithm, as particle's inertia, ipar $=0.95$, as self-adjustment weight, selw $=0.05$, and as social-adjustment weight, $\operatorname{soc} w=0.02$; and (iii) for the HS algorithm, as new population size, $N e w_{-} P o p=40$, as harmony memory consideration rate, $H M C R=0.9$, as pitch adjustment rate, $P A R=0.3$, and as bandwidth, $b w=\frac{1}{100}\left(\boldsymbol{\theta}^{\boldsymbol{u}}-\boldsymbol{\theta}^{\boldsymbol{l}}\right)$.

In this manner, to consider the impact of the residual weights on the updated value of the single-objective function, $f(\boldsymbol{\theta})$, a trial-and-error criterion [5] was considered. Table 3 shows the eleven cases established in terms of the different values considered for the residuals associated with the natural frequencies, $\sum w_{j}^{f}$, and vibration modes., $\sum w_{j}^{m}$, respectively. Additionally, each case was run ten times in order to take into account that the initial population has been generated randomly.

As result of the updating process, Table 3 shows the average values of the residuals for the three considered NIC algorithms in terms of the different value considered for the weighting factors. Additionally, Fig. 6a illustrates the representation in the multi-objective functional space of the residuals associated with each case and computational algorithm.

Table 3. Average values of the residuals in terms of the weighting factors under the SOF approach for the three considered NIC algorithms (GA, PS and HS).

Additionally, the simulation time required to solve the updating problem for each case has been determined. Thus, the following average simulation time were computed: (i) $74072 \mathrm{~s}$ for the GA; (ii) $43857 \mathrm{~s}$ for the PS; and (iii) $34380 \mathrm{~s}$ for the HS.

According to these results, two main conclusions can be drawn: (i) the accuracy of the three mentioned NIC algorithm is similar; and (ii) the HS algorithm allows reducing the simulation time required to perform the updating process. In this manner, the HS algorithm allows performing the FE model updating of this structure more efficiently than the other algorithms since it reduces the computational cost without compromising the accuracy of the adjustment.

\section{Comparison among GA, PS and HS for the FE Model Updating under the MOF Approach}

Subsequently, the initial FE model of the laboratory footbridge was also updating under the MOF approach considering the three mentioned computational algorithms. The updating process was performed again via its implementation in both the FE analysis package Ansys [33] and the mathematical package Matlab [37]. As comparison criteria, the two above mentioned were considered again.

For the three NIC algorithms under the MOF approach, the same design variables (common and particular) were considered. Additionally, the number of elements of the Pareto front was established in 25. The updating problem was solved ten times for each NIC algorithms to take into account the random generation of the initial population.

As result of the updating process, Fig. $6 \mathrm{~b}$ illustrates the average Pareto front obtained for each considered NIC algorithm. 
Fig. 6. Comparison among the different residuals in the functional space considering the three NIC algorithms (GA, PS and HS): a) The SOF approach; b) The MOF approach; and c) Both the SOF and MOF approaches.

Additionally, the simulation time required, to obtain the Pareto front corresponding to each mentioned NIC algorithm, has been determined. Thus, the following average simulation times were computed: (i) $307057 \mathrm{~s}$ for the GA; (ii) $240663 \mathrm{~s}$ for the PS; and (iii) $121676 \mathrm{~s}$ for the HS.

According to these results, three main conclusions can be drawn: (i) the accuracy of both the PS and HS algorithm is greater than the one provided by the GA since the Pareto front obtained by these two algorithms allows reducing the overall value of the residuals between the numerical and experimental modal properties of the structure; (ii) the accuracy between the PS and HS algorithms is similar; and (iii) the HS algorithm allows reducing again the simulation time required to perform the updating process. In this manner, the HS algorithm is again the most efficient NIC algorithm to solve the updating problem under the MOF approach.

\section{Comparison between the SOF Approach and the MOF Approach for the FE Model Updating of the Laboratory Footbridge}

Otherwise the performance of the two approaches has been compared. For this purpose, three comparison criteria were taken into account: (i) the sweep capacity; (ii) the computational cost; and (iii) the goodness of the solution.

Fig. 6c illustrates a comparison among the solutions obtained considering the two approach (SOF and MOF) and the three mentioned NIC algorithms (GA, PS and HS). The pair of residuals of each solution has been represented in the multi-objective functional space.

As Fig. 6c shows although all the solutions obtained under the SOF approach belong to the Pareto front of the updating problem, however they focus on one of the branches of the curve. Thus, the sweep capacity of the SOF approach is lower than the one obtained under the MOF approach. Additionally, the set of solutions obtained under the SOF approach present a worse adjustment than the set of solutions obtained under the MOF approach. All the solutions obtained under the SOF approach present a greater distance to the coordinate origin (overall adjustment error) and they are far from the socalled knee-point, or solutions which better balances the value of the two types of residuals [24]. Additionally, the simulation time required to perform the updating process under the MOF approach is lower than the one required under the SOF approach. Thus, the most efficient algorithm, the HS algorithms, takes about $378180 \mathrm{~s}$ to determine the best updated model under the SOF while it only takes $121677 \mathrm{~s}$ under the MOF approach (121676 s to determine the Pareto front and $1 \mathrm{~s}$ to solve the decisionmaking problem).

For all these reasons, it can be established that the MOF approach of the HS algorithm is the best option to perform the FE model updating of a civil engineering structure under the MLM.

Finally, for the sake of completeness, the modal properties of the footbridge, after the updating process, are shown in Table 4 and Fig. 7. The best solution of the Pareto front provided by the HS algorithm under the MOF approach has been considered for this purpose. This best solution has been obtained via the implementation of the NBI method [24] on this Pareto front. Thus, Table 4 shows the first seven updated natural frequencies, $f_{u p d, j}(\boldsymbol{\theta})$, of the structure and Fig. 7 illustrates the first 
seven vibration modes, $\phi_{u p d, j}(\boldsymbol{\theta})$. As Table 4 shows, the correlation between the updated and experimental modal properties of the footbridge, after the updating process, is good. All the relative differences, $\Delta f_{\text {exp,j }}^{u p d, j}(\boldsymbol{\theta})$, are lower than $5 \%$, and all the $\operatorname{MAC}(\boldsymbol{\theta})_{\text {exp,j }}^{\text {upd,j }}$ ratios are greater than 0.9 .

Fig. 7. Comparison between the updated, $\phi_{u p d, j}(\boldsymbol{\theta})$, and experimental, $\phi_{\text {exp }, j}$, vibration modes of the laboratory footbridge (being $j$ the considered vibration mode).

Table 4. Correlation between the updated and experimental modal properties of the laboratory footbridge (being $f_{u p d, j}(\boldsymbol{\theta})$ the updated natural frequencies; $f_{\text {exp,j }}$ the experimental natural frequencies; $\Delta \mathrm{f}_{\mathrm{exp}, \mathrm{j}}^{\mathrm{upd}, \mathrm{\theta}}(\boldsymbol{\theta}$, the relative differences between the updated and experimental natural frequencies; and the $\operatorname{MAC}(\boldsymbol{\theta})_{\mathrm{exp}, \mathrm{j}}^{\mathrm{upd}, \mathrm{j}}$ ratio of the $j$ considered vibration mode).

\section{Conclusions}

FE model updating of civil engineering structures is usually performed under the MLM. According to this method, the FE model updating problem may be formulated as an optimization problem. Thus, the main objective of this optimization problem is to obtain the value of the most relevant physical parameters of the structure which minimizes the relative differences between the numerical and experimental modal properties of the structure. As two modal properties are normally considered, the natural frequencies and their associated vibration modes, one key aspect to take into account is how to handle the influence of the residuals associated with each modal property in the solution of the updating problem. In order to overcome this drawback, two approaches have been proposed to formulate the optimization problem: (i) the SOF approach; and (ii) the MOF approach.

Additionally, due to both the complexity of the models and the non-linear relationship between the residuals and the considered design parameters, computational intelligence algorithms are have been extensively used to solve the updating problem. Among these computational algorithms, NIC algorithms have been shown especially effective to cope with this problem. Nevertheless, their use presents a main drawback, the high simulation time required to solve this optimization problem.

In order to shed light to these two drawbacks, this study has two main objectives: (i) to establish which is the most adequate approach to formulate the updating problem under the MLM; and (ii) to determine which is the most efficient NIC algorithm to solve the FE model updating problem among three different computational algorithms (GA, PS and HS).

For this purpose, the FE model updating of a real civil engineering structures has been performed under the two mentioned approaches and considering the three mentioned NIC algorithm. As benchmark structure, a laboratory steel footbridge located at the University of Exeter (U.K.) has been considered. A FE model of the structure has been built. The numerical modal properties of the footbridge have been obtained from a FE modal analysis. Subsequently, the experimental modal properties of the footbridge were obtained by the signal processing (experimental modal analysis) of the records obtained during a forced vibration test. The correlation between the numerical and experimental modal properties has been computed and the necessity of improving the FE model has been revealed. Different comparison criteria have been regarded to compare the results of the different FE model updating processes. Thus, for the 
establishment of the most adequate approach: (i) the sweep capacity; (ii) the computational cost; and (iii) the goodness of the solution have been taken into account. Additionally, for the determination of the most efficient NIC algorithm: (i) the computational cost; and (ii) the accuracy of the adjustment have been considered.

As result of this study two main conclusions have been obtained: (i) the MOF approach is the most adequate option to perform the FE model updating of civil engineering structures under the MLM since it allows sweeping more widely the space search; it allows reducing the overall simulation time required to solve the updating problem and it allows obtaining an updated model which better balances the influence of the two considered residuals; and (ii) the HS algorithm is the most efficient NIC algorithm among the three considered ones to solve the updating problem since it allows reducing clearly the computational cost without compromising the accuracy of the adjustment.

In this manner, the results provided herein can be implemented directly to improve the FE model updating of complex civil engineering structures for practical engineering applications. Nevertheless, further studies are recommended to validate the performance of the HS algorithm when it is implemented for the FE model updating under the MOF approach of different types of civil engineering structures.

\section{References}

[1] Friswell MI, Mottershead JE. Finite Element Model Updating in Structural Dynamics. Kluwer Academic Publishers, 1995.

[2] Brownjohn JMW, Xia P-Q, Hao H, Sia Y. (2001). Civil structure condition assessment by FE model updating: methodology and case studies. Finite Elem. Anal. Des. 2001; 37: 761-775. doi: https://doi.org/10.1016/S0168$\underline{874 X(00) 00071-8 .}$.

[3] Bucher C, Pham HA. On model updating of existing structures utilizing measured dynamic responses. Struct. Infrastruct Eng. 2005; 1(2): 135-143, doi: http://dx.doi.org/10.1080/15732470412331289297.

[4] Liu T, Zhang Q, Zordan T, Briseghella B. Finite Element Model Updating of Canonica Bridge Using Experimental Modal Data and Genetic Algorithm. Struct. Eng. Int. 2016; 26(1): 27-36. doi: https://doi.org/10.2749/101686616X14480232444405.

[5] Teughels A, Maeck J, De Roeck G. Damage assessment by FE model updating using damage functions. Comput. Struct. 2002; 80(25): 1869-1879. doi: https://doi.org/10.1016/S0045-7949(02)00217-1.

[6] Mordini A, Savov K, Wenzel H. The Finite Element Model Updating: A Powerful Tool for Structural Health Monitoring. Struct. Eng. Int. 2007; 17(4): 352-358. doi: https://doi.org/10.2749/101686607782359010.

[7] Chen B, Zhu Y, Hu J, Principe JC. System Parameter Identification. Information Criteria and Algorithms. Tsinghua University Press Ltd. Published by Elsevier Inc 2013. doi: https://doi.org/10.1016/B978-0-12-404574-3.00008-7.

[8] Marwala T. Finite-Element-Model Updating Using Computational Intelligence Techniques. Applications to Structural Dynamics. Springer, London, 2010.

[9] Chen Z. Bayesian filtering: From Kalman filters to particle filters, and beyond. Stat. 2003; 182: 1-69. doi: https://doi.org/10.1080/02331880309257.

[10] Rao CR, Toutenburg H, Fieger A. Linear Models and Generalizations: Least Squares and Alternatives. $2^{\text {nd }}$ ed. Springer; 2007. 
[11] Jiménez-Alonso JF, Sáez A. Model Updating for the Selection of an Ancient Bridge Retrofitting Method. Struct. Eng. Int. 2016; 26(1): 17-26, doi: https://doi.org/10.2749/101686615X14355644771333.

[12] Marwala T; Boulkaibet I; Adhikari S. Probabilistic Finite Element Model Updating Using Bayesian Statistics: Applications to Aeronautical and Mechanical Engineering. John Wiley \& Sons, London, 2016.

[13] Val DV, Stewart MG. Assessment of Ageing Reinforced Concrete StructuresCurrent Situation and Future Challenges. Struct. Eng. Int. 2009; 19(2): 211-219, doi: https://doi.org/10.2749/101686609788220114.

[14] Mottershead JE, Link M, Friswell MI. The sensitivity method in finite element model updating: a tutorial. Mech. Syst. Signal Pr. 2011; 25(7): 2275-2296, doi: https://doi.org/10.1016/j.ymssp.2010.10.012.

[15] Maia NMM, Silva JMM. Theoretical and Experimental Modal Analysis. Research studies Press Ltd., Somerset, England, 1997.

[16] Jin S, Cho S, Jung H, Lee J, Yun C A new multi-objective approach to finite element model updating. J. Sound Vib. 2014; 333(11): 2323-2338. doi: https://doi.org/10.1016/j.jsv.2014.01.015.

[17] Park W, Kim H-K, Jongchil P. Finite Element Model Updating for a Cable-Stayed Bridge Using Manual Tuning and Sensitivity-Based Optimization. Struct. Eng. Int. 2012; 22(1): 14-19, doi: https://doi.org/10.2749/101686612X13216060212870.

[18] Kim G-H, Park Y-S. An improved updating parameter selection method and finite element model update using multi-objective optimisation technique. Mech. Syst. Signal Pr. 2004; 18(1): 59-78, doi: https://doi.org/10.1016/S08883270(03)00042-6.

[19] Ghee CK, Perry MC. Structural Identification and Damage Detection using Genetic Algorithms p. 140. CRC Press, Taylor and Francis Group, London, 2010.

[20] Kennedy J, Eberhart R. Particle swarm optimization, in Proceedings of ICNN'95 International Conference on Neural Networks. 1995; 4, 1942-1948. doi: http://dx.doi.org/10.1109/ICNN.1995.488968.

[21] Geem ZW, Kim JH, Loganathan GV. A new heuristic optimization algorithm: harmony search. Simul. 2001; 76(2): 60-68. doi: https://doi.org/10.1177/003754970107600201.

[22] Saka MP, Aydogdu I, Hasancebi O, Geem ZW. Harmony Search Algorithms in Structural Engineering. In: Yang XS, Koziel S (eds) Computational Optimization and Applications in Engineering and Industry. Studies in Computational Intelligence, vol 359. Springer, Berlin, Heidelberg, 2011. doi: https://doi.org/10.1007/978-3-642-20986-4_6.

[23] Hudson E, Reynolds P. Design and Construction of a Reconfigurable Pedestrian Structure. Exp. Techniques. 2017; 41(2): 203-214. doi: https://doi.org/10.1007/s4079.

[24] Deb, K, Gupta, S. Understanding knee points in bicriteria problems and their implications as preferred solution principles. Eng. Optimiz. 2011; 43(11): 11751204. doi: https://doi.org/10.1080/0305215X.2010.548863.

[25] Infantes M, Naranjo-Pérez J, Sáez A, Jiménez-Alonso JF. Determining the best Pareto-solution in a multi-objective approach for model updating. IABSE Symposium, Guimaraes 2019: Towards a Resilient Built Environment Risk and Asset Management - Report, pp. 523-530, 2019. 
[26] Costa C, Ribeiro D, Jorge P, Silva R, Arêde A, Calçada R. Calibration of the numerical model of a stone masonry railway bridge. Eng. Struct. 2016; 123: 345-371. doi: https://doi.org/10.1016/i.engstruct.2016.05.044.

[27] Zivanovic S, Pavic A, Reynolds P. Finite element modelling and updating of a lively footbridge: The complete process. Eng. Struct 2007; 301(1-2): 126-145. doi: http://dx.doi.org/10.1016/j.jsv.2006.09.024.

[28] Deb K, Pratap A, Agarwal S, Meyarivan T. A fast and elitist multi-objective genetic algorithm: NSGA-II. IEEE Trans. Evol. Comput. 2002; 6(2): 182-197. doi: http://dx.doi.org/10.1109/4235.996017.

[29] Srinivas N, Deb K. Multi-objective optimization using nondominated sorting in genetic algorithms. IEEE Trans. Evol. Comput. 1994; 2(3): 221-248. doi: http://dx.doi.org/10.1162/evco.1994.2.3.221.

[30] Coello CAC and Lechuga MS (2002). A proposal for multiple objective particle swarm optimization, in Proceedings of the 2002 Congress on Evolutionary Computation. CEC'02 doi: http://dx.doi.org/10.1109/CEC.2002.1004388.

[31] Geem ZW. Multi-objective optimization of time-cost trade-off using harmony search. J. Constr. Eng. M. 2009; 136(6): 711-716. doi: https://doi.org/10.1061/(ASCE)CO.1943-7862.0000167.

[32] SPS (2019). Sandwich Plate System heavy engineering composite from Intelligent Engineering n.d. http:/www.ie-sps.com. (accessed May 2019).

[33] Ansys Mechanical Release 19.0. (2019). n.d. www.ansys.com. (accessed May 2019).

[34] Eurocode 3. Design of Steel Structures. CEN, 2005.

[35] Clark JL. Structural Design of Polymer Composites-EUROCOMP Design Code and Handbook, E\&FN Spon, 1996.

[36] Fox R, Kapoor M. Rates of change of eigenvalues and eigenvectors. AIAA J. 1968; 6: 2426-2429. doi: https://doi.org/10.2514/3.5008.

[37] Matlab R2019a (2019), n.d. http://www.mathworks.com. (accessed May 2019). 
Table 1. Correlation between the initial numerical and experimental modal properties of the laboratory footbridge (being $f_{n u m, j}(\boldsymbol{\theta})$ the numerical natural frequencies; $f_{\text {exp }, j}$ the experimental natural frequencies; $\Delta f_{\text {exp,j }}^{n u m}(\boldsymbol{\theta})$, the relative differences between the numerical and experimental natural frequencies; and the $\operatorname{MAC}(\boldsymbol{\theta})_{\text {exp,j }}^{\text {num } j}$ ratio of the $j$ considered vibration mode).

\begin{tabular}{|c|c|c|c|c|}
\hline Vibration Mode & $f_{\text {num }, j}(\boldsymbol{\theta})[\mathrm{Hz}]$ & $f_{\text {exp,j }}[\mathrm{Hz}]$ & $\Delta f_{\text {exp,j }}^{\text {num }}(\boldsymbol{\theta})[\%]$ & $M A C(\boldsymbol{\theta})_{\text {exp }, j}^{\text {num }}[-]$ \\
\hline $1\left(^{\text {st }}\right.$ Bending $)$ & 3.638 & 3.810 & -4.509 & 0.999 \\
\hline $2\left(1^{\text {st }}\right.$ Torsional $)$ & 5.329 & 5.144 & 3.600 & 0.994 \\
\hline $3\left(2^{\text {nd }}\right.$ Torsional $)$ & 10.185 & 8.485 & 20.033 & 0.990 \\
\hline $4\left(2^{\text {nd }}\right.$ Bending $)$ & 11.310 & 12.366 & -8.540 & 0.877 \\
\hline $5\left(3^{\text {rd }}\right.$ Bending $)$ & 17.364 & 18.605 & -6.670 & 0.985 \\
\hline $6\left(3^{\text {rd }}\right.$ Torsional $)$ & 20.238 & 20.459 & -1.080 & 0.993 \\
\hline $7\left(^{\text {th }}\right.$ Bending $)$ & 21.105 & 22.980 & -8.159 & 0.910 \\
\hline
\end{tabular}


Table 2. Search domain (lower bound, $\boldsymbol{\theta}^{\mathbf{l}}$, and upper bound, $\boldsymbol{\theta}^{\mathbf{u}}$ ) of the considered physical parameters of the model, $\boldsymbol{\theta}$.

\begin{tabular}{|c|l|c|c|}
\hline $\begin{array}{c}\text { Physical } \\
\text { Parameter, } \boldsymbol{\theta} .\end{array}$ & $\begin{array}{c}\text { Definition } \\
\text { (Fig. 7) }\end{array}$ & $\begin{array}{c}\text { Lower } \\
\text { Bound, } \boldsymbol{\theta}^{\boldsymbol{l}} \text {. }\end{array}$ & $\begin{array}{c}\text { Upper } \\
\text { Bound, } \boldsymbol{\theta}^{\boldsymbol{u}}\end{array}$ \\
\hline$\theta_{1}$ & Young's modulus steel. Sections 1/12 [MPa] & $1.89 \cdot 10^{5}$ & $2.31 \cdot 10^{5}$ \\
\hline$\theta_{2}$ & Young's modulus steel. Sections 2/11 [MPa] & $1.89 \cdot 10^{5}$ & $2.31 \cdot 10^{5}$ \\
\hline$\theta_{3}$ & Young's modulus steel. Sections 3/10 [MPa] & $1.89 \cdot 10^{5}$ & $2.31 \cdot 10^{5}$ \\
\hline$\theta_{4}$ & Young's modulus steel. Sections 4/9 [MPa] & $1.89 \cdot 10^{5}$ & $2.31 \cdot 10^{5}$ \\
\hline$\theta_{5}$ & Young's modulus steel. Sections 5/8 [MPa] & $1.89 \cdot 10^{5}$ & $2.31 \cdot 10^{5}$ \\
\hline$\theta_{6}$ & Young's modulus steel. Sections 6/7 [MPa] & $1.89 \cdot 10^{5}$ & $2.31 \cdot 10^{5}$ \\
\hline$\theta_{7}$ & Young's modulus polyurethane [MPa] & $0.75 \cdot 10^{3}$ & $1.50 \cdot 10^{3}$ \\
\hline$\theta_{8}$ & Young's modulus steel of the bolts [MPa] & $2.10 \cdot 10^{5}$ & $2.10 \cdot 10^{6}$ \\
\hline$\theta_{9}$ & Longitudinal stiffness spring [N/m] & $1.38 \cdot 10^{7}$ & $1.10 \cdot 10^{8}$ \\
\hline$\theta_{10}$ & Lateral stiffness spring [N/m] & $4.75 \cdot 10^{6}$ & $3.80 \cdot 10^{7}$ \\
\hline
\end{tabular}


Table 3. Average values of the residuals in terms of the weighting factors under the single-objective function approach (SOF) for the three considered NIC algorithms (GA, PS and HS).

\begin{tabular}{|c|c|c|c|c|c|c|c|c|}
\hline Case & $\sum$ & $\sum$ & $w_{j}^{f}$ & $\sum$ & $w_{j}^{m}$ & \multicolumn{2}{|c|}{ GA $\left[x 10^{-3}\right]$} & \multicolumn{2}{c|}{ PS $\left[x 10^{-3}\right]$} & \multicolumn{2}{c|}{ HS $\left[x 10^{-3}\right]$} \\
\cline { 4 - 9 } & & & $\sum r_{j}^{f}(\boldsymbol{\theta})^{2}$ & $\sum r_{j}^{m}(\boldsymbol{\theta})^{2}$ & $\sum r_{j}^{f}(\boldsymbol{\theta})^{2}$ & $\sum r_{j}^{m}(\boldsymbol{\theta})^{2}$ & $\sum r_{j}^{f}(\boldsymbol{\theta})^{2}$ & $\sum r_{j}^{m}(\boldsymbol{\theta})^{2}$ \\
\hline 01 & 1.0 & 0.0 & 3.63 & 2.69 & 3.56 & 2.68 & 3.55 & 2.69 \\
\hline 02 & 0.9 & 0.1 & 3.57 & 2.66 & 3.56 & 2.68 & 3.55 & 2.69 \\
\hline 03 & 0.8 & 0.2 & 3.59 & 2.62 & 3.58 & 2.51 & 3.58 & 2.66 \\
\hline 04 & 0.7 & 0.3 & 3.58 & 2.53 & 3.57 & 2.52 & 3.61 & 2.52 \\
\hline 05 & 0.6 & 0.4 & 3.57 & 2.56 & 3.57 & 2.50 & 3.62 & 2.50 \\
\hline 06 & 0.5 & 0.5 & 3.58 & 2.51 & 3.58 & 2.51 & 3.58 & 2.57 \\
\hline 07 & 0.4 & 0.6 & 3.58 & 2.52 & 3.58 & 2.49 & 3.62 & 2.51 \\
\hline 08 & 0.3 & 0.7 & 3.59 & 2.48 & 3.58 & 2.49 & 3.63 & 2.51 \\
\hline 09 & 0.2 & 0.8 & 3.58 & 2.51 & 3.58 & 2.49 & 3.62 & 2.49 \\
\hline 10 & 0.1 & 0.9 & 3.58 & 2.53 & 3.59 & 2.47 & 3.70 & 2.47 \\
\hline 11 & 0.0 & 1.0 & 3.59 & 2.50 & 3.59 & 2.49 & 3.64 & 2.46 \\
\hline
\end{tabular}


Table 4. Correlation between the updated and experimental modal properties of the laboratory footbridge (being $f_{u p d, j}(\boldsymbol{\theta})$ the updated natural frequencies; $f_{\text {exp,j }}$ the experimental natural frequencies; $\Delta \mathrm{f}_{\mathrm{exp}, \mathrm{j}}^{\mathrm{upd}}(\boldsymbol{\theta})$, the relative differences between the updated and experimental natural frequencies; and the $\operatorname{MAC}(\boldsymbol{\theta})_{\mathrm{exp}, \mathrm{j}}^{\mathrm{upd}, \mathrm{j}}$ ratio of the $j$ considered vibration mode).

\begin{tabular}{|c|c|c|c|c|}
\hline Vibration Mode & $f_{u p d, j}(\boldsymbol{\theta})[\mathrm{Hz}]$ & $f_{\text {exp }, j}[\mathrm{~Hz}]$ & $\Delta f_{e x p, j}^{u p d, j}(\boldsymbol{\theta})[\%]$ & $M A C(\boldsymbol{\theta})_{e x p, j}^{u p d, j}$ \\
\hline $1\left(1^{\text {st }}\right.$ Bending $)$ & 3.854 & 3.810 & 1.155 & 0.999 \\
\hline $2\left(1^{\text {st }}\right.$ Torsional $)$ & 5.489 & 5.144 & 4.907 & 0.994 \\
\hline $3\left(2^{\text {nd }}\right.$ Torsional $)$ & 8.365 & 8.485 & -1.414 & 0.988 \\
\hline $4\left(2^{\text {nd }}\right.$ Bending $)$ & 11.896 & 12.366 & -3.800 & 0.905 \\
\hline 5 (3rd Bending) & 18.662 & 18.605 & 0.306 & 0.987 \\
\hline $6\left(3^{\text {rd }}\right.$ Torsional $)$ & 20.016 & 20.459 & -2.163 & 0.993 \\
\hline 7 (4 $4^{\text {th }}$ Bending) & 22.506 & 22.980 & -2.063 & 0.957 \\
\hline
\end{tabular}




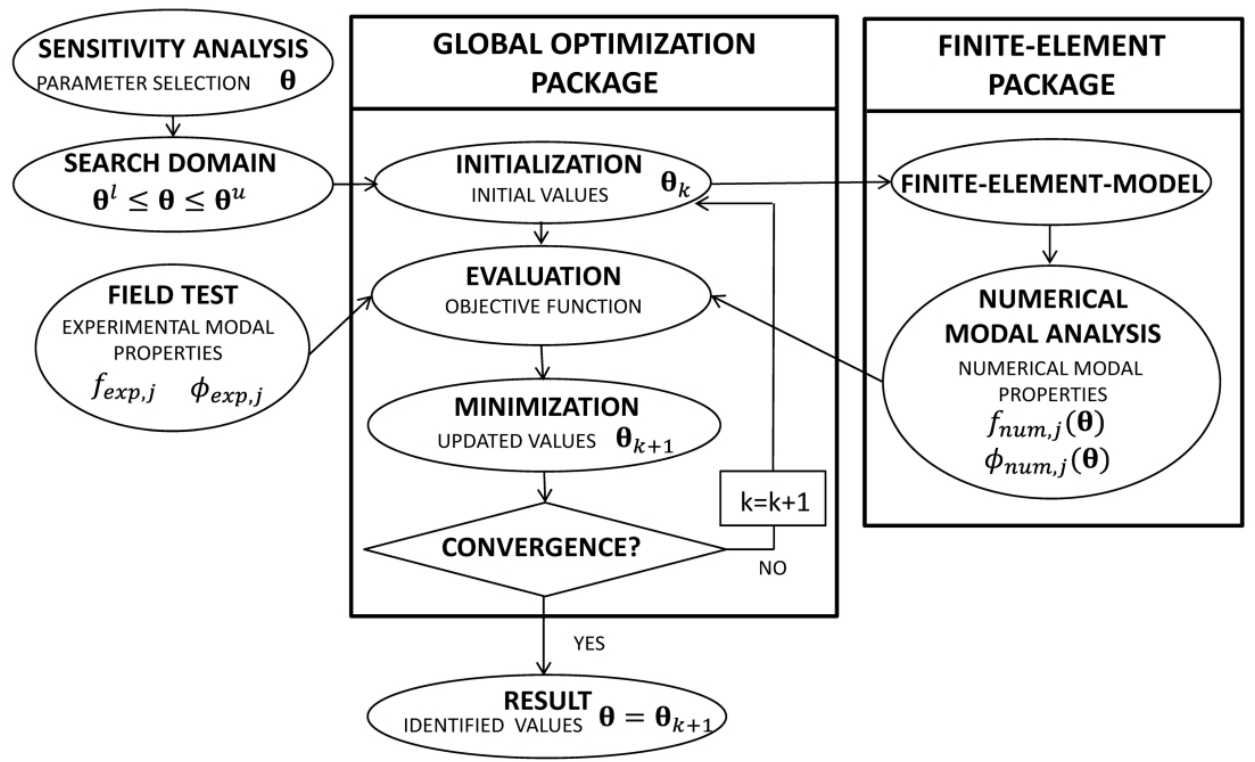

Figure 1

$147 \times 90 \mathrm{~mm}(600 \times 600$ DPI $)$ 


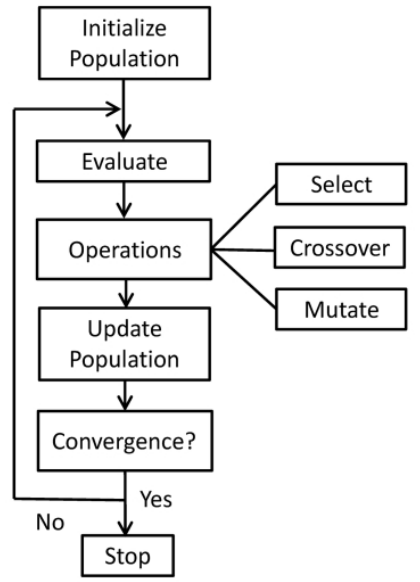

a) $\mathrm{GA}$

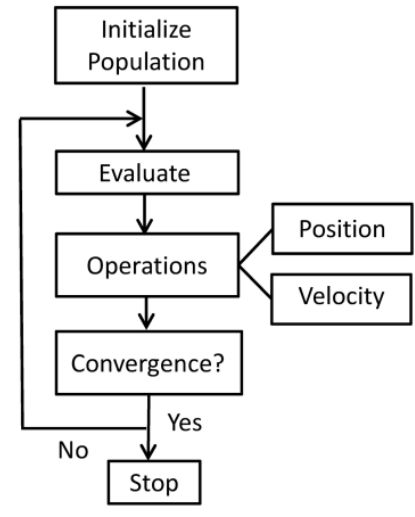

b) PS

Figure 2

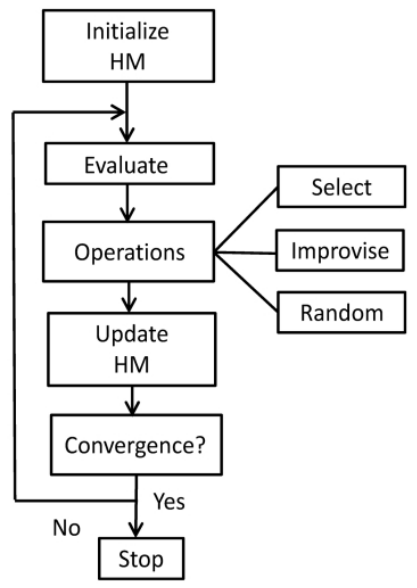

c) HS

$156 \times 87 \mathrm{~mm}(600 \times 600 \mathrm{DPI})$ 


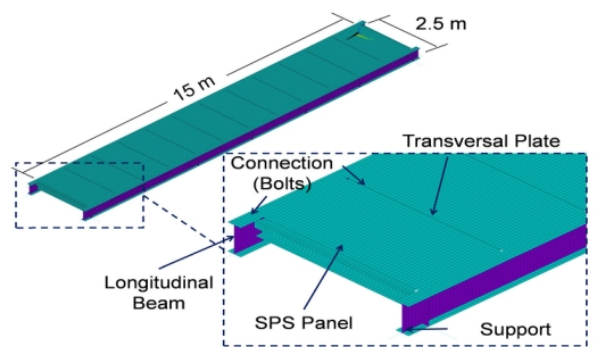

Laboratory Footbridge

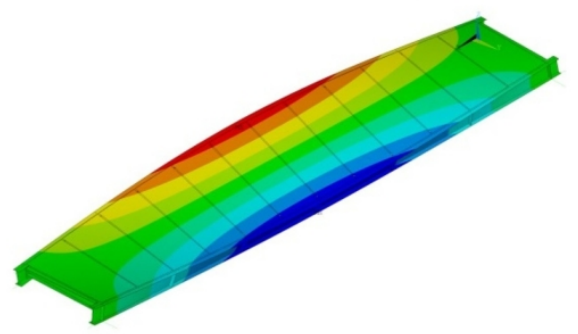

$$
f_{\text {num }, 2}(\boldsymbol{\theta})=5.329 \mathrm{~Hz}
$$
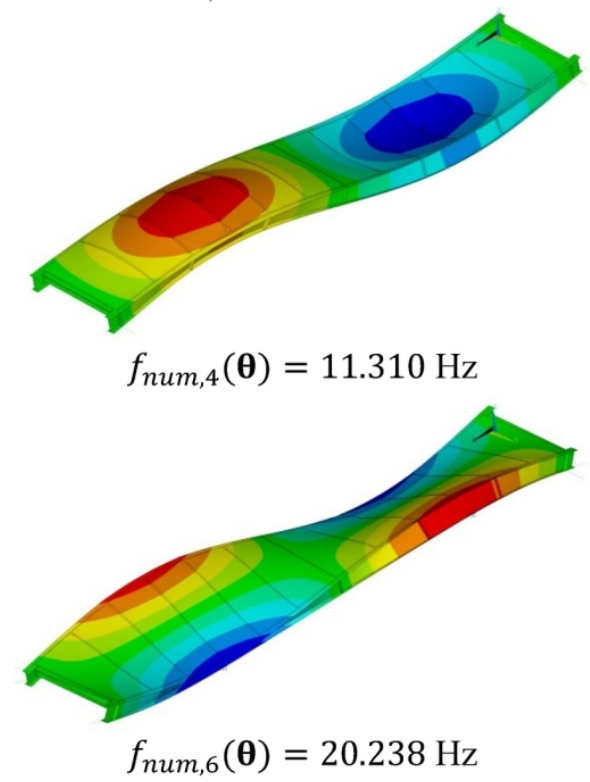
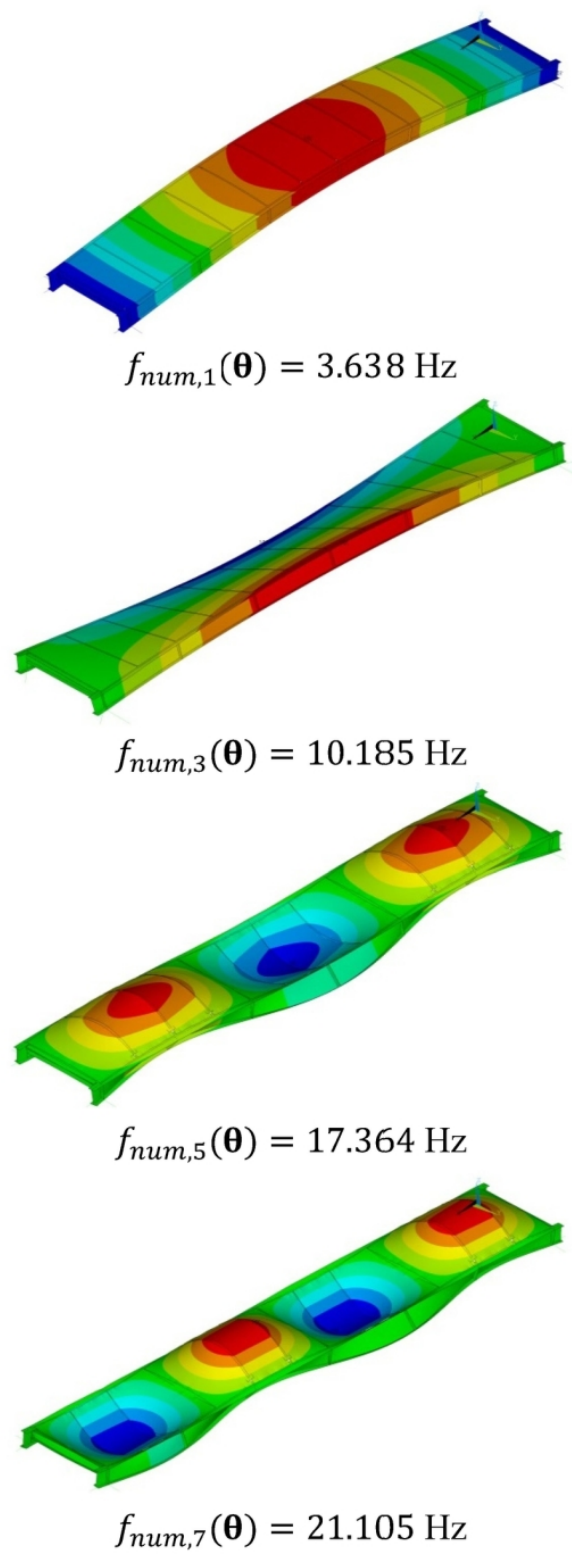

Figure 3

$145 \times 182 \mathrm{~mm}(300 \times 300 \mathrm{DPI})$ 

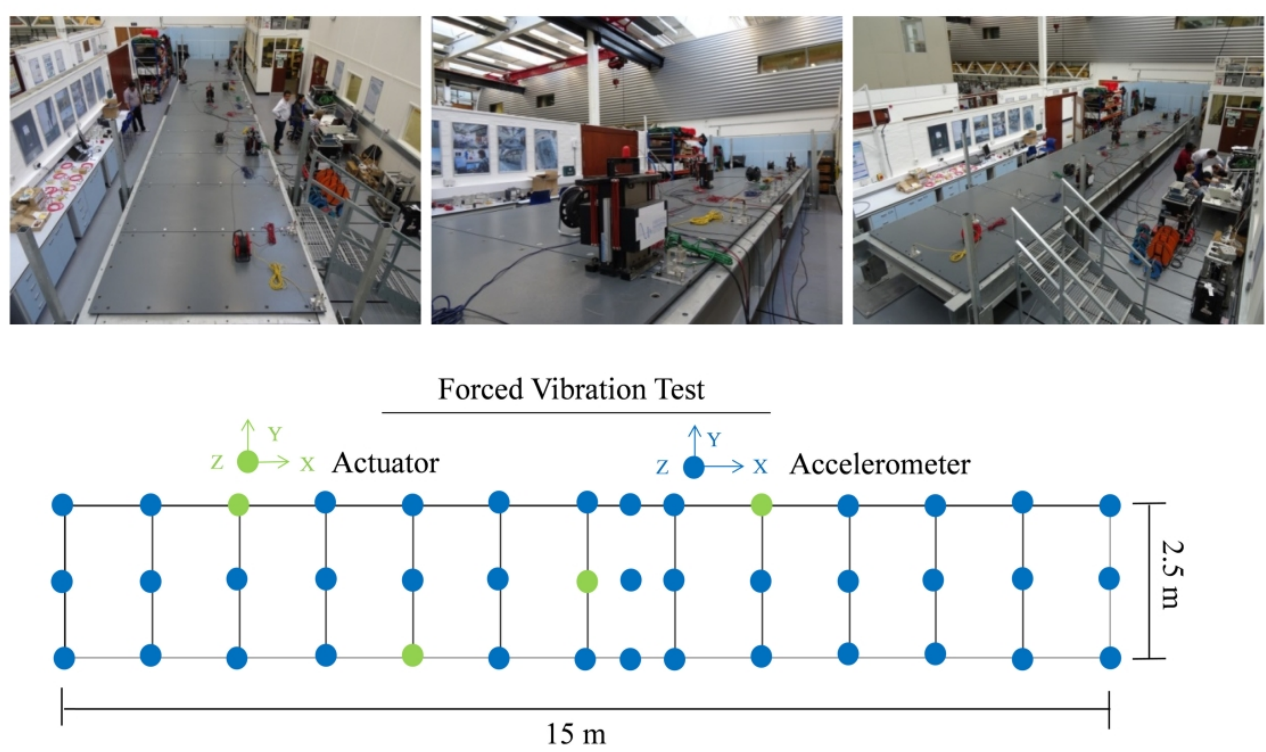

Figure 4

$158 \times 91 \mathrm{~mm}(600 \times 600 \mathrm{DPI})$ 

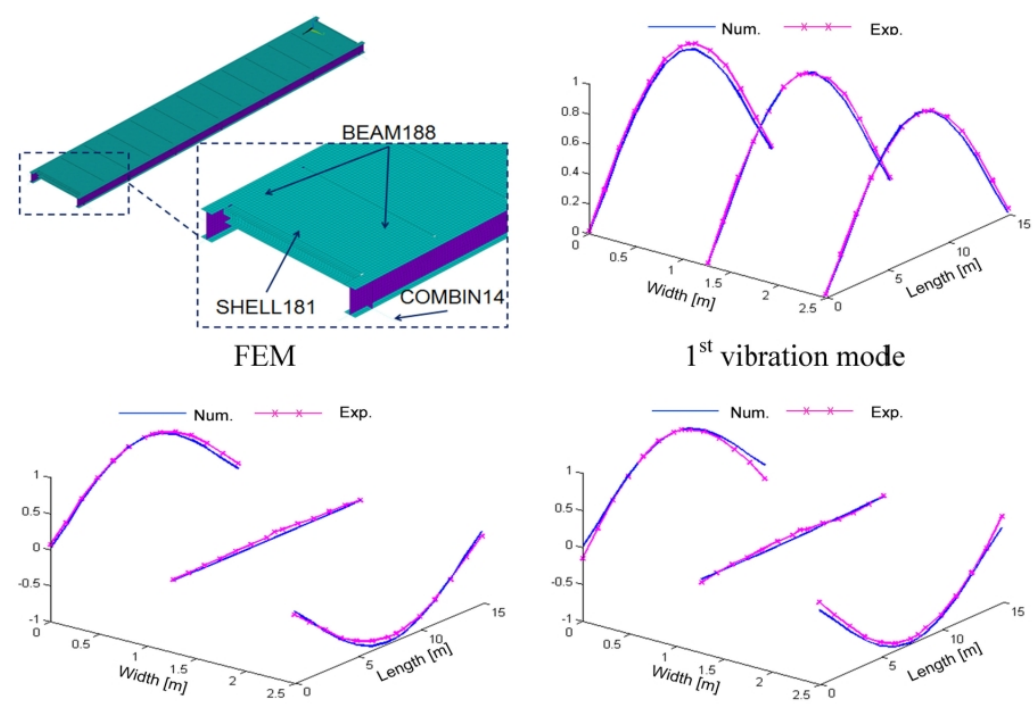

$1^{\text {st }}$ vibration mode

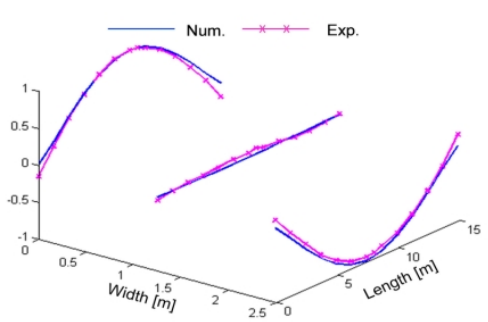

$2^{\text {nd }}$ vibration mode

$3^{\text {rd }}$ vibration mode
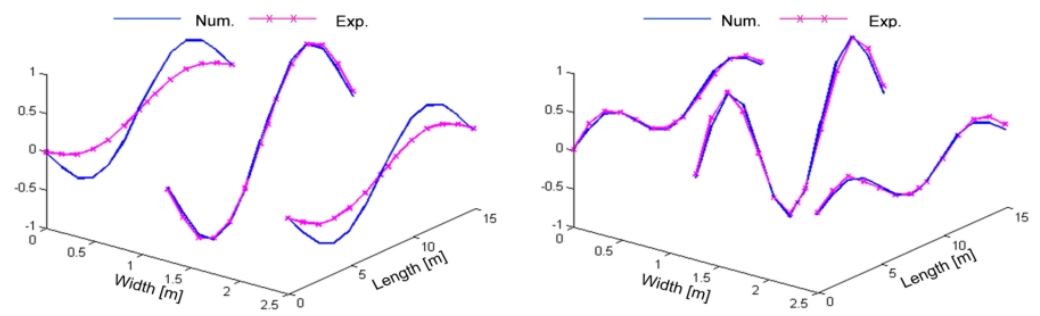

$4^{\text {th }}$ vibration mode

$5^{\text {th }}$ vibration mode
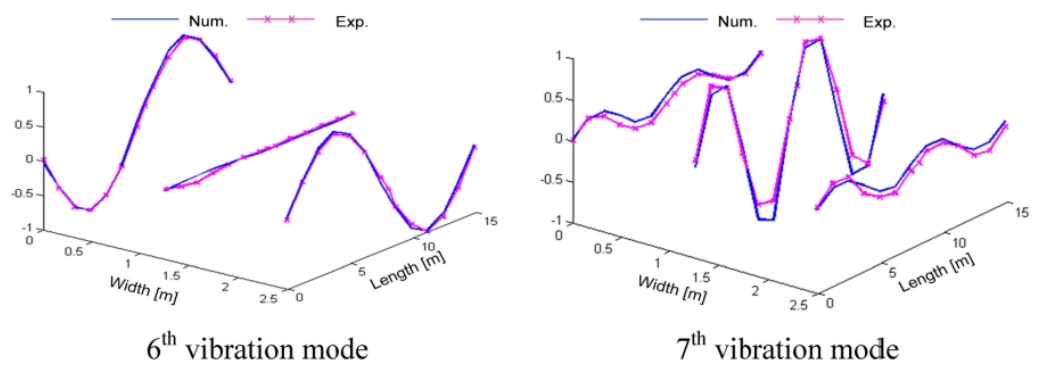

Figure 5

$149 \times 217 \mathrm{~mm}(300 \times 300 \mathrm{DPI})$ 
b)

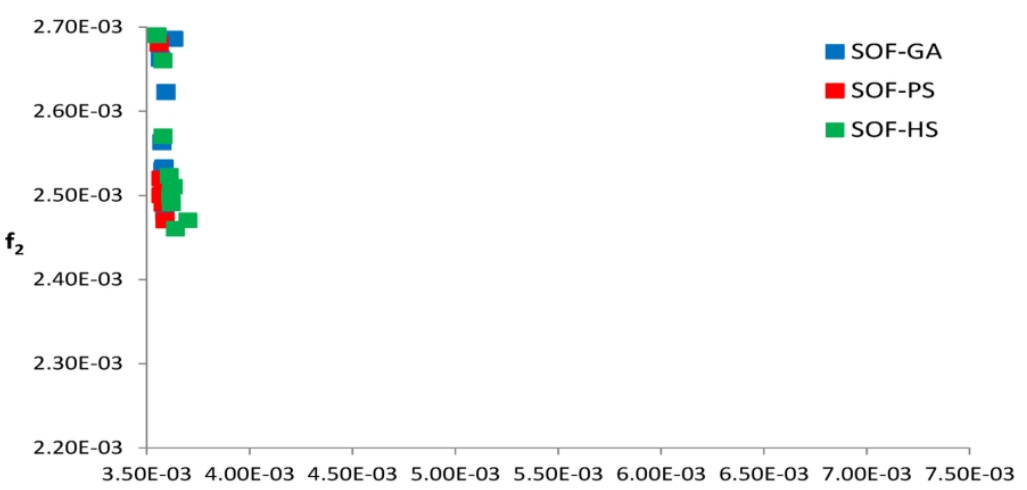

a) $f_{1}$

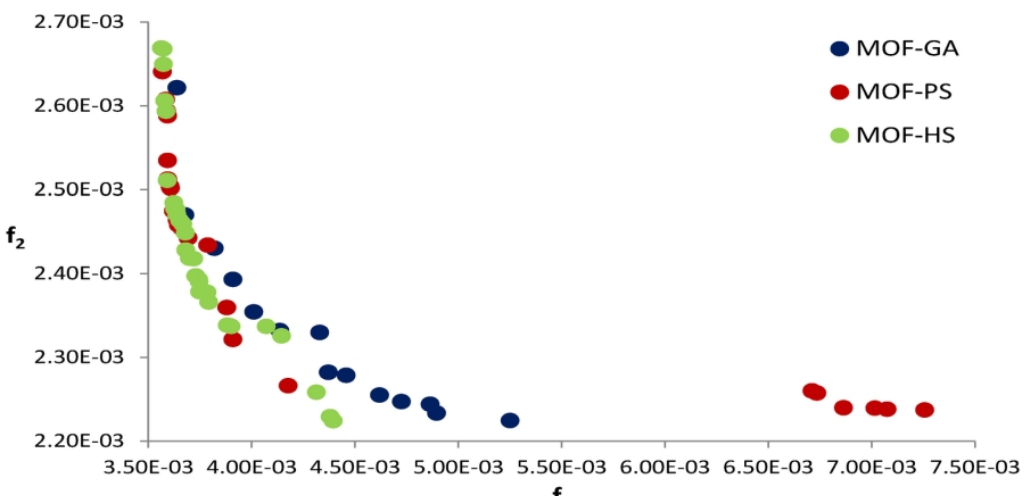
(

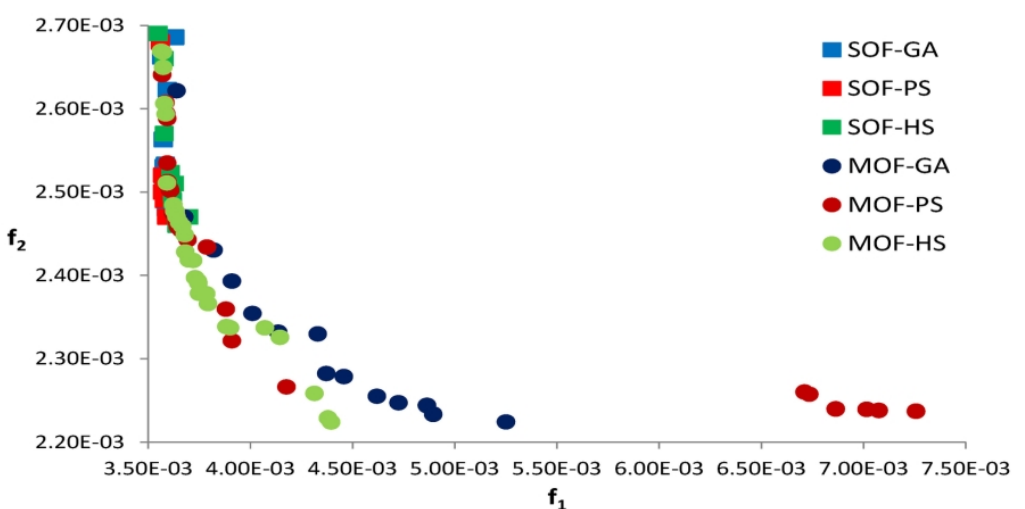

Figure 6

$151 \times 212 \mathrm{~mm}(300 \times 300 \mathrm{DPI})$ 

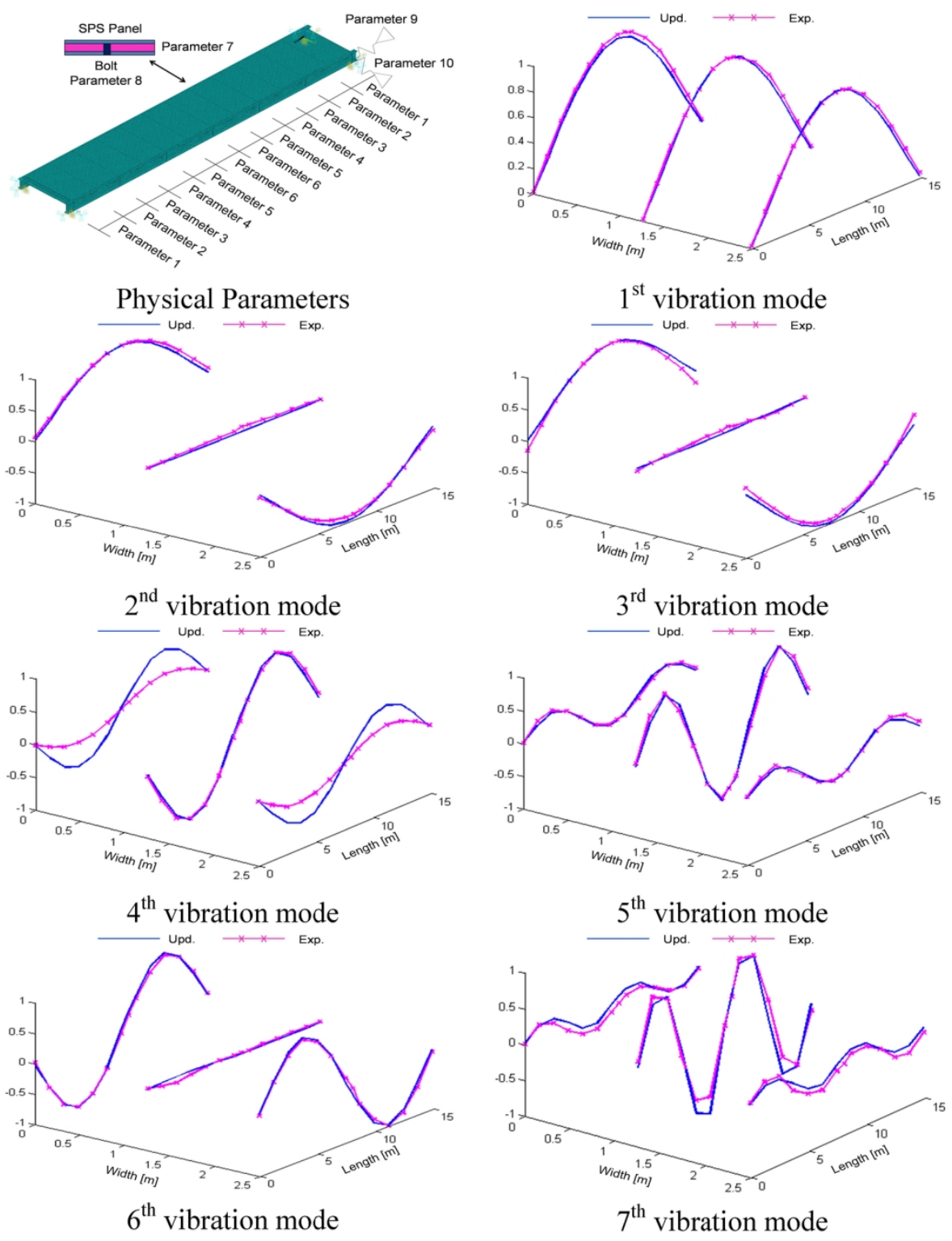

Figure 7

$144 \times 184 \mathrm{~mm}(300 \times 300$ DPI) 